\title{
Lipid A Has Significance for Optimal Growth of Coxiella burnetii in Macrophage-Like THP-1 Cells and to a Lesser Extent in Axenic Media and Non-phagocytic Cells
}

\author{
Tao Wang ${ }^{1}$, Yonghui Yu ${ }^{1}$, Xiaofei Liang ${ }^{2}$, Shengdong Luo ${ }^{1}$, Zemin He ${ }^{1}$, Zhihui Sun ${ }^{1}$, \\ Yongqiang Jiang ${ }^{1}$, Anders Omsland ${ }^{3}$, Pei Zhou ${ }^{4}$ and Lihua Song ${ }^{1 *}$

\begin{abstract}
${ }^{1}$ State Key Laboratory of Pathogen and Biosecurity, Beijing Institute of Microbiology and Epidemiology, Beijing, China, ${ }^{2}$ Department of Chemistry, Duke University, Durham, NC, United States, ${ }^{3}$ Paul G. Allen School for Global Animal Health, College of Veterinary Medicine, Washington State University, Pullman, WA, United States, ${ }^{4}$ Department of Biochemistry, Duke University Medical Center, Durham, NC, United States
\end{abstract}

\section{OPEN ACCESS}

Edited by: Jan Potempa,

University of Louisville, United States

Reviewed by:

Edward Shaw,

Oklahoma State University,

United States

Michael F. Minnick,

University of Montana, United States

*Correspondence:

Lihua Song

songlihua@gmail.com

Received: 10 February 2018 Accepted: 22 May 2018

Published: 08 June 2018

Citation:

Wang T, Yu Y, Liang X, Luo S, He Z, Sun Z, Jiang $Y$, Omsland A, Zhou $P$ and Song $L$ (2018) Lipid A Has

Significance for Optimal Growth of Coxiella burnetii in Macrophage-Like THP-1 Cells and to a Lesser Extent in Axenic Media and Non-phagocytic Cells.

Front. Cell. Infect. Microbiol. 8:192. doi: 10.3389/fcimb.2018.00192
Lipid A is an essential basal component of lipopolysaccharide of most Gram-negative bacteria. Inhibitors targeting $\mathrm{LpxC}$, a conserved enzyme in lipid A biosynthesis, are antibiotic candidates against Gram-negative pathogens. Here we report the characterization of the role of lipid A in Coxiella burnetii growth in axenic media, monkey kidney cells (BGMK and Vero), and macrophage-like THP-1 cells by using a potent LpxC inhibitor -LPC-011. We first determined the susceptibility of $C$. burnetii LpxC to LPC-011 in a surrogate $E$. coli model. In E. coli, the minimum inhibitory concentration (MIC) of LPC-011 against $C$. burnetii $\mathrm{LpxC}$ is $<0.05 \mu \mathrm{g} / \mathrm{mL}$, a value lower than the inhibitor's MIC against $E$. coli $\mathrm{LpxC}$. Considering the inhibitor's problematic pharmacokinetic properties in vivo and Coxiella's culturing time up to 7 days, the stability of LPC-011 in cell cultures was assessed. We found that regularly changing inhibitor-containing media was required for sustained inhibition of $C$. burnetii $\mathrm{LpxC}$ in cells. Under inhibitor treatment, Coxiella has reduced growth yields in axenic media and during replication in non-phagocytic cells, and has a reduced number of productive vacuoles in such cells. Inhibiting lipid A biosynthesis in $C$. burnetii by the inhibitor was shown in a phase II strain transformed with chlamydial $k d t A$. This exogenous KdtA enzyme modifies Coxiella lipid A with an $\alpha-K d o-(2 \rightarrow 8)-\alpha-K d o$ epitope that can be detected by anti-chlamydia genus antibodies. In inhibitor-treated THP-1 cells, Coxiella shows severe growth defects characterized by poor vacuole formation and low growth yields. Coxiella progenies prepared from inhibitor-treated cells retain the capability of normally infecting all tested cells in the absence of the inhibitor, which suggests a dispensable role of lipid A for infection and early vacuole development. In conclusion, our data suggest that lipid A has significance for optimal development of Coxiella-containing vacuoles, and for robust multiplication of $C$. burnetii in macrophage-like THP-1 cells. Unlike many bacteria, C. burnetii replication in axenic media and non-phagocytic cells was less dependent on normal lipid A biosynthesis.

Keywords: Q fever, Coxiella burnetii, LpxC inhibitor, LPC-011, lipid A, LPS 


\section{INTRODUCTION}

Coxiella burnetii is a geographically widely distributed, Gramnegative intracellular bacterium. It is the causative agent of $Q$ fever which may manifest in humans as an acute disease (mainly as a self-limiting febrile illness, pneumonia, or hepatitis) or as a chronic disease (mainly endocarditis in patients with previous valvulopathy) (Maurin and Raoult, 1999). The majority ( $50-60 \%)$ of human infections are asymptomatic (Maurin and Raoult, 1999; Hechemy, 2012). Resolution of symptoms does not mean the patient is clear of infection (Harris et al., 2000). Chronic infections are rare but can be fatal if untreated. C. burnetii is a significant cause of culture-negative endocarditis in the United States (Mulye et al., 2017). Treatment of chronic infections is challenging and currently requires a combined antibiotic therapy with doxycycline and hydroxychloroquine for a minimum of 18 months (Angelakis and Raoult, 2010).

C. burnetii is the only known bacterium that replicates within acidified, degradative phagolysosome-like vacuoles (termed Coxiella-containing vacuole, or CCV) of eukaryotic cells. Similar to Chlamydia spp., it has two morphologically distinct cell types that comprise a biphasic developmental cycle (Waag, 2007). A small cell variant (SCV), likely the extracellular survival form, invades the host and develops into a large cell variant (LCV) for replication. The LCV replicates and its progenies differentiate back into SCVs during the stationary phase of the organism's growth cycle. Both the SCV and LCV forms of Coxiella are infectious (Wiebe et al., 1972; Minnick and Raghavan, 2012).

Gram-negative bacteria contain a principle component called lipopolysaccharide (LPS) in the outer leaflet of the outer membrane. LPS protects Gram-negative bacteria against external damaging agents such as antibiotics and detergents. It consists of a membrane saccharolipid called lipid A, a core oligosaccharide, and a distal repeating polysaccharide units (Raetz et al., 2007). Lipid A is essential for growth of most Gram-negative bacteria, and its biosynthetic pathway is an attractive target for the development of novel antibiotics (Barb and Zhou, 2008; Zhou and Zhao, 2017). Diverse inhibitors targeting LpxC, an enzyme responsible for the first committed step in lipid A biosynthesis, have been synthesized (Kalinin and Holl, 2017). These inhibitors represents a class of promising antibiotic candidates, and are new tools for studying biosynthesis and function of lipid A or LPS in Gram-negative bacteria (Nguyen et al., 2011; Tomaras et al., 2014).

Virulent C. burnetii harbors LPS like other Gram-negative bacteria, but undergoes an irreversible modification of its LPS, termed phase variation, when extensively passaged in immunoincompetent hosts. The phase variation is a transition of C. burnetii from a virulent phase I to an avirulent phase II state (Hackstadt, 1988). LPS from phase I C. burnetii contains two unique biomarkers of methylated sugars (virenose and dihydrohydroxystreptose) at its O-specific chain, while LPS from phase II C. burnetii is severely truncated and only contains lipid A and partial core oligosaccharide. LPS from phase I C. burnetii may mask toll-like receptor ligands from innate immune recognition by human dendritic cells, thus might play an important role in C. burnetii persistent infections (Shannon et al., 2005). Before the advent of axenic culture (Omsland et al., 2009) and modern genetic techniques in C. burnetii (Beare and Heinzen, 2014), the investigation of biology and pathogenesis of C. burnetii LPS mutants was limited to the use of naturally occurring LPS mutants (Narasaki and Toman, 2012; Larson et al., 2016). Thus far LPS is the only C. burnetii virulence factor evaluated in immunocompetent rodent models (mice and guinea pigs) (Moos and Hackstadt, 1987; Andoh et al., 2007).

Lipid A is the basal component of LPS, and is termed endotoxin due to its role in LPS toxicity. The lipid A moiety plus one 3-deoxy-D-manno-oct-2-ulosonic acid (Kdo) is the minimal requirement for survival of many Gram-negative bacteria. In Chlamydia trachomatis, also an intracellular vacuole pathogen, lipid A is essential for the generation of infectious progeny (Nguyen et al., 2011). C. burnetii lipid A is well characterized (Narasaki and Toman, 2012). Its proximal region contains three Kdo residues-one of which is suggested to be a Kdo-like substance, likely a Ko (modified from Kdo by a KdoO enzyme) (Toman and Skultety, 1994; Chung and Raetz, 2011; Narasaki and Toman, 2012). Endotoxin activities of C. burnetii lipid A were up to 1,000-fold less active than lipid A of E. coli (Amano et al., 1987). Though various biological and immunomodulatory functions of C. burnetii LPS have been described, the importance of lipid A in C. burnetii biology remains unclear.

In this study, we characterized the role of lipid $A$ in C. burnetii growth in different culture systems by using a potent LpxC inhibitor -LPC-011. We first evaluated the minimum inhibitory concentration (MIC) of LPC-011 against C. burnetii LpxC in a surrogate $E$. coli model. A reliable condition of effectively inhibiting lipid A biosynthesis in C. burnetii was then established in a transformant carrying Chlamydia trachomatis $k d t A$. Inhibitory effects of LPC-011 against C. burnetii were tested with phase I and phase II strains. Our data suggest that lipid A partially contributes to the formation of productive Coxiellacontaining vacuoles in non-phagocytic cells, and has significance for C. burnetii robust multiplication in macrophage-like THP1 cells, but to a much lesser extent in axenic media and nonphagocytic cells. Our data suggest that LpxC inhibitors have potential as anti-C. burnetii agents during infection.

\section{MATERIALS AND METHODS}

\section{Cell Lines, Bacterial Strains, and Key Reagents}

African monkey kidney cells (Vero) and Buffalo green monkey kidney cells (BGMK) were grown in high-glucose-containing Dulbecco's Modified Eagle Medium (DMEM) supplemented with $10 \%$ FBS. Human monocytic leukemia cells (THP-1) were cultured in RPMI 1640 medium supplemented with 10\% FBS. C. burnetii Nine Mile phase II, Henzerling phase I and Chlamydia trachomatis $\mathrm{B} / \mathrm{QH} 111 \mathrm{~L}$ are from our laboratory strain collection. All media and FBS were purchased from Life Technologies (Beijing, China). Synthesis of the LpxC inhibitor, LPC-011, was described previously (Liang et al., 2011). PCR primers used in this study are shown in Table S1. 


\section{Construction of an E. coli Mutant Carrying C. burnetii IpxC With an Intron Insertion in the Chromosomal Copy of IpxC}

A DNA fragment including sequences flanking C. burnetii lp $x C$ (CBU_0142) was amplified from C. burnetii Nine Mile phase II genomic DNA with primers CBlpxCF and CBlpxCR, and was cloned into a $\mathrm{T}$ vector of pZeroBack/Blunt (TIANGEN Biotech, Beijing, China). The resulting plasmid pCBlpxC was transformed into E. coli BL21(DE3). The chromosomal copy of E. coli $l p x C$ was disrupted by insertion of a group II intron using a commercial TargeTron gene knockout system (TA0100, Sigma-Aldrich). The target site for TargeTron insertion was between nucleotide residues 326 and 327 on the sense strand. Four PCR primers -IBS1/2, EBS1/delta, EBS2, and EBS/Universal, were used to modify the intron for insertion into the predicted site according to the TargeTron system user guide (sigma-aldrich.com/targetron).

\section{Construction of a Shuttle Vector for Lipid A-Modification in C. burnetii}

The C. burnetii shuttle vector pMMGKkdtA was constructed by cloning kanamycin resistance $\left(\mathrm{Kan}^{\mathrm{R}}\right)$ cassette, eGFP and C. trachomatis $k d t A$ gene into RSF1010 ori-based vector pMMB207, kindly provided by Xuehong Zhang (Shanghai Jiaotong University, Shanghai, China). The $\mathrm{Kan}^{\mathrm{R}}$, eGFP and $k d t A$ genes were driven by $C$. burnetii promoters of CBU1169, CBU0311 and CBU1169, respectively. Promoter regions of CBU0311 and CBU1169 were amplified with P311Kpn1F/P311-R and P1169-F/P1169-R, respectively. The $\mathrm{Kan}^{\mathrm{R}}$ and eGFP genes were amplified from pEASY-T1 and pEGFP-C1, with Kan-F/Kan-XhoI-R and eGFP-F/eGFP-R, respectively. Overlapping PCR with primers P311-KpnI-F/Kan-XhoI-R was used to produce the P311-eGFP-P1169-Kan fragment. The CBU1169 promoter region and the $C$. trachomatis $k d t A$ gene were amplified with P1169-NheI-F/P1169-kdtA-R and kdtA-NheI-F/kdtA-XhoI-R, respectively, and then overlapping PCR with primers P1169-NheI-F/kdtA-XhoI-R to generate the P1169-kdtA segment. The kdtA-P1169-P311-eGFP-P1169-Kan fragment was then amplified with primers kdtA-XhoI-R/KanKpnI-R. The RSF1010 ori-contained fragment was amplified from pMMB207 with p207-XhoI-F/p207-KpnI-R. The two fragments were digested with $\mathrm{KpnI}$ and $\mathrm{XhoI}$, and ligated to generate the vector pMMGKkdtA (Datasheet S1). The resulting vector was transformed into C. burnetii Nine Mile phase II by electroporation (Beare and Heinzen, 2014). C. burnetii transformants were cloned three successive times in ACCM-2 plates and were expanded in BGMK cells (Beare and Heinzen, 2014).

\section{Burnetii Culture and Purification}

All C. burnetii strains (Nine Mile phase II, Henzerling phase I and transformants of Nine Mile phase II) were purified from BGMK cultures by centrifugation through a 30\% Renografin density gradient. Their progenies generated under LpxC inhibitor treatment were purified from BGMK cultures with regularly changed cell media containing $2 \mu \mathrm{g} / \mathrm{mL}$ LPC-011 every $48 \mathrm{~h}$. Purified organisms were suspended in SPG $(0.25 \mathrm{M}$ sucrose,
$10 \mathrm{mM}$ sodium phosphate, $5 \mathrm{mM} \mathrm{L}$-glutamic acid), and kept frozen at $-80^{\circ} \mathrm{C}$. For testing the inhibitory effects of LPC-011 in axenic culture, ACCM-2 medium plus $500 \mu \mathrm{M}$ tryptophan was used (Vallejo Esquerra et al., 2017).

\section{Activity Assessment of LPC-011 in Spent Media}

Spent media in cell culture or axenic culture of C. burnetii were collected at indicated time points, then were centrifuged at $20,000 \mathrm{~g}$ for $10 \mathrm{~min}$ to remove bacteria or cell debris and kept frozen at $-80^{\circ} \mathrm{C}$. To assess the activity of LPC-011, E. coli TOP 10 was added into $1 \mathrm{~mL}$ spent media in $1.5 \mathrm{~mL}$ tubes and cultured overnight at $37^{\circ} \mathrm{C}$ with shaking $(220 \mathrm{rpm})$. The optical density at $550 \mathrm{~nm}\left(\mathrm{OD}_{550}\right)$ was measured. All samples were performed in triplicate.

\section{C. burnetii Infections and Phase Contrast Microscopy}

Monolayers of cells (Vero, BGMK, or THP-1) were grown in appropriate media as described above in 24-well plates and were infected with parent $C$. burnetii strains or their progenies generated under LPC-011 treatment at appropriate MOIs. Before infecting THP- 1 cells, cultures were treated with $200 \mathrm{nM}$ phorbol 12-myristate 13 -acetate (PMA) for $24 \mathrm{~h}$ for differentiation into macrophage-like cells as previously described (Larson et al., 2015). To facilitate infection, plates were centrifuged at $800 \mathrm{~g}$ for $30 \mathrm{~min}$, then was washed with PBS and $1 \mathrm{ml}$ RPMI 1640 medium supplemented with 5\% FBS was added per well. Medium not supplemented with FBS was used for the duration of BGMK infections. All infections were performed in triplicate. Plates were incubated at $37^{\circ} \mathrm{C}$. Phase-contrast images were captured at a magnification of $\times 400$ by using a Nikon eclipse TS100 inverted microscope to record the vacuole morphology of C. burnetii strains.

\section{Immunofluorescence}

Cells grown on coverslips were infected with C. burnetii as described above. At 7 days post-infection, cells were fixed with ice-cold methanol for $10 \mathrm{~min}$ at room temperature. After washing with PBS, cells were subjected to antibody and chemical staining. Human sera from chronic $\mathrm{Q}$ fever patients plus an anti-human IgG conjugated with Alexa Fluor 488 were used to visualize C. burnetii. Lipid A of C. burnetii transformants carrying C. trachomatis $k d t A$ was stained with mouse antiChlamydia genus monoclonal antibodies (Santa Cruz Biotech) and a rabbit anti-mouse IgG-PE conjugate (Life Technologies). An Olympus FV1000S laser confocal microscope (Olympus) was used to analyze the stained samples.

\section{Quantitative PCR}

Purified C. burnetii recovered from host cell and axenic cultures were quantified by using $\mathrm{qPCR}$ as previously described with minor modifications (Coleman et al., 2004). Cell samples were harvested by using trypsin (Hyclone) treatment and centrifugation. Total DNA was extracted with DNeasy Blood Tissue Kit (Qiagen). Genome copy numbers were determined by Taqman probe qPCR specific to dotA by using an ABI 7300 sequence detection system (Applied Biosystems). PCR conditions 
were as follows: initial denaturation at $94^{\circ} \mathrm{C}$ for $10 \mathrm{~min}$, followed by 40 cycles of amplification at $94^{\circ} \mathrm{C}$ for $15 \mathrm{~s}, 60^{\circ} \mathrm{C}$ for $1 \mathrm{~min}$.

\section{Transmission Electron Microscopy}

Cell were fixed with $0.1 \mathrm{M}$ phosphates $/ 2.5 \%$ glutaraldehyde buffer $(\mathrm{pH}=7.4)$ for $4 \mathrm{~h}$, then incubated in $1 \%$ cacodylatebuffered osmic acid and treated with $1 \%$ uranyl acetate in distilled water. Samples were dehydrated with graded amounts of acetone and embedded in Spurr's resin. Ultrathin sections were stained with $1 \%$ uranyl acetate and Reynold's lead citrate. Images were captured with a Hitachi H-7600 transmission electron microscope (Hitachi).

\section{Statistical Analysis}

A two-tailed Student $t$ test was used for qPCR analysis of C. burnetii growth yields under various conditions. The standard deviation was determined from three independent biological replicates.

\section{RESULTS}

\section{A Single High Dose of LpxC Inhibitors Cannot Restrict C. burnetii Growth in Vero Cells}

Various cell lines have been used for culture and antibiotic susceptibility testing of C. burnetii (Angelakis and Raoult, 2010). When extensively passaged in cell cultures, full-length LPS of phase I C. burnetii tends to become severely truncated while lipid A remains unaffected, suggesting selective pressure helps to retain the lipid A component of LPS. Because lipid A is essential for the viability of most Gram-negative bacteria, we tested the effectiveness of a broad-spectrum LpxC inhibitor -LPC-011 as a potential anti-Coxiella agent. In preliminary experiments, Vero cells were infected using a high MOI ( $>50)$ of phase I and phase II C. burnetii strains by centrifugation at $800 \times \mathrm{g}$ for $30 \mathrm{~min}$, and fresh media containing different doses $(0,1-10 \mu \mathrm{g} / \mathrm{mL})$ of LPC011 were added after infection. Vacuole formation and bacterial growth were assessed by phase contrast microscopy for over 7 days. Unexpectedly, no perceptible phenotypes were observed during cell cultures of $C$. burnetii, although similar cell toxicity was observed at high concentrations of LPC-011 (Nguyen et al., 2011), suggesting lipid A could be dispensable for C. burnetii culture in Vero cells. The C. burnetii culture in the presence or absence of $2 \mu \mathrm{g} / \mathrm{mL}$ LPC-011 is shown in Figure 1. However, a conclusive role of the essentiality of lipid A in C. burnetii growth cannot be determined due to the lack of a method to detect the disruption of lipid A biosynthesis. To measure the potential MIC of LPC-011 against $C$. burnetii during intracellular replication is further complicated by the pathogen's lengthy developmental cycle inside acidic vacuoles, and the known difference of MICs of LPC-011 for intracellular vs. extracellular growth of the same bacterium such as Salmonella typhimurium (Nguyen et al., 2011).

\section{LPC-011 Inhibits Coxiella LpxC at $0.05 \mu \mathrm{g} / \mathrm{mL}$ in a Surrogate E. coli System}

LPC-011 is reportedly a broad-spectrum inhibitor of LPS biosynthesis against a variety of Gram-negative pathogens.

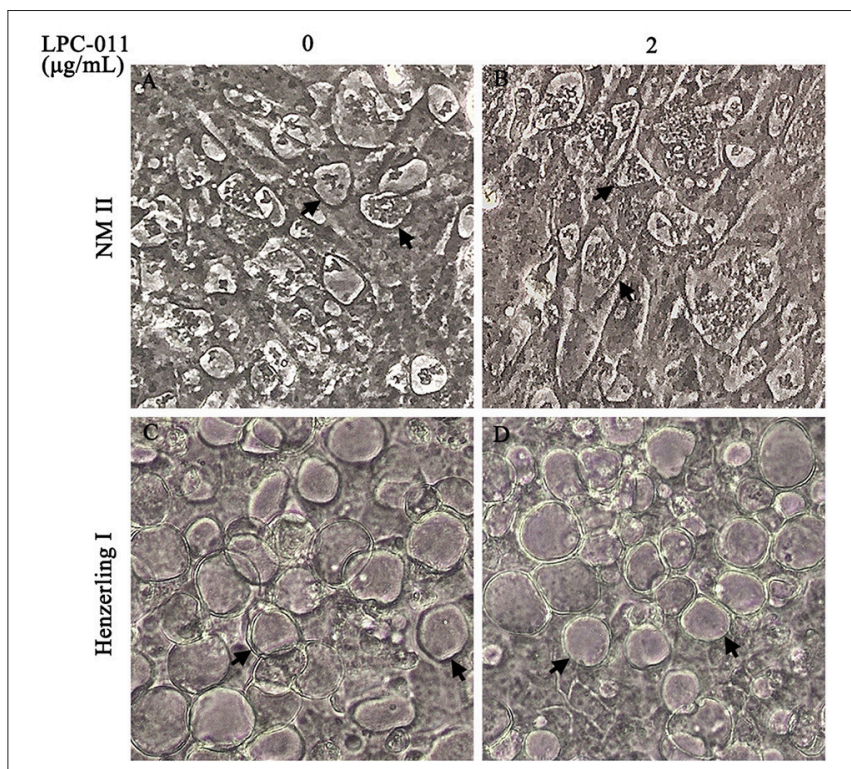

FIGURE 1 | C. burnetii CCVs appear normal on Vero cells with a single dose $(2 \mu \mathrm{g} / \mathrm{mL})$ of LPC-011. Monolayers of $C$. burnetii Nine Mile phase II $(\mathbf{A}, \mathbf{B})$ and Henzerling phase I (C,D) infected cells with or without adding LPC-011 were imaged by phase microscopy at 7 days post-infection. Arrows indicate CCVs.

Indeed, the MIC of LPC-011 against E. coli W3110 is as low as $0.03 \mu \mathrm{g} / \mathrm{mL}$ (Liang et al., 2011). We next sought to determine the susceptibility of C. burnetii LpxC to LPC-011 in a surrogate E. coli model (Figure 2), as it has been shown that susceptibility of $E$. coli to LpxC inhibitors is dependent on the source of $l p x C$ gene (Lee et al., 2011; Liang et al., 2011). The C. burnetii LpxC shares $55 \%$ amino acid identity with the E. coli LpxC, including eight identical amino acids at the active site (Coggins et al., 2003; Whittington et al., 2003). E. coli BL21(DE3) was transformed with a plasmid carrying C. burnetii $l p x C$, and the chromosomal $l p x C$ was inactivated by inserting a group II intron. The successful disruption of $E$. coli $l p x C$ suggests that $C$. burnetii $l p x C$ can complement the essential function of the endogenous $E$. coli lpxC in trans. In LB plates containing $0.05 \mu \mathrm{g} / \mathrm{mL}$ of LPC-011, the E. coli mutant complemented with C. burnetii $1 p x C$ failed to grow while growth of the wild type $E$. coli was not affected, suggesting LPC-011 has an MIC against C. burnetii LpxC below $0.05 \mu \mathrm{g} / \mathrm{mL}$. Importantly this MIC is lower than the MIC against E. coli LpxC -between 0.05 and $0.1 \mu \mathrm{g} / \mathrm{mL}$. Compared to the previous reported MIC $(0.03 \mu \mathrm{g} / \mathrm{mL})$ of LPC-011 against E. coli W3110 using liquid cultures (Liang et al., 2011), our measured MIC value of LPC-011 against E. coli BL21(DE3) is slightly higher, which may reflect the difference of assaying methods (liquid culture vs. plating).

\section{Reduced Growth of C. burnetii in Axenic Medium Containing $5 \mu \mathrm{g} / \mathrm{mL}$ LPC-011}

ACCM-2 supplemented with tryptophan is an acidified citrate cysteine medium that was recently modified for improved culture of C. burnetii (Omsland et al., 2009, 2011; Vallejo Esquerra et al., 

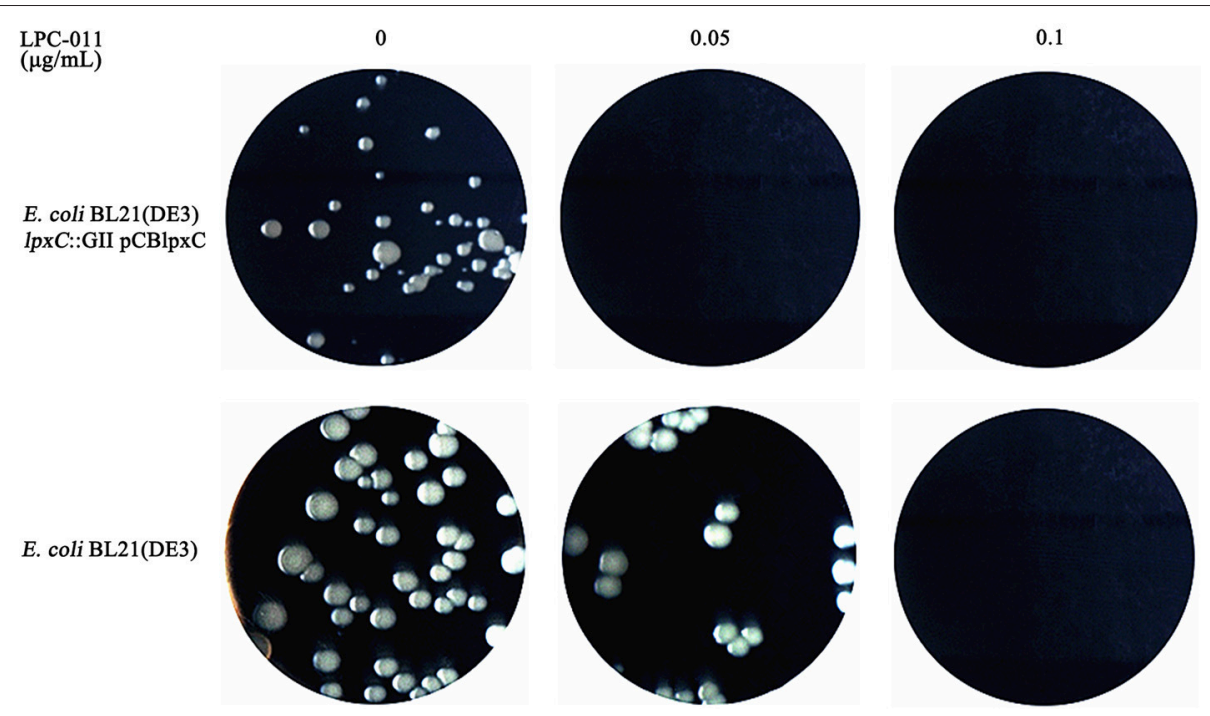

FIGURE 2 | Growth comparison of wild type E. coli and an E. coli mutant carrying C. burnetii IpxC on LB-agar plates containing different doses of LPC-011. The E. coli mutant (E. coli BL21(DE3) IpxC::Gll pCBIpxC) was constructed by inserting a group II intron in the chromosomal lpxC gene of an E. coli transformant carrying the $\mathrm{pCB} \mid \mathrm{pxC}$ plasmid, which contains $C$. burnetii IpxC.

2017). It can support 3-4 logs of growth of C. burnetii after 7 days of cultivation in $5 \% \mathrm{O}_{2}$ at $37^{\circ} \mathrm{C}$. We tested if a single dose $(5 \mu \mathrm{g} / \mathrm{mL},>100 \times$ MIC) of LPC-011 affects C. burnetii growth in ACCM-2 plus tryptophan. Under control conditions, C. burnetii phase I and phase II strains normally grew 2.2 and 3.2 logs, compared to 2.0 and 2.1 logs of increase with LPC-011 treatment, respectively (Figure 3A, Table S2). Between the two strains, the growth of the phase II strain was more affected by the inhibitor (Table S2). The growth of E. coli in spent ACCM-2 was used to assess the activity of LPC-011 (Figure 3B). The spent ACCM-2 with LPC-011 maintained an E. coli-lethal activity, while the spent ACCM-2 without LPC011 appeared to affect $E$. coli growth differentially. In the absence of LPC-011, compared to the phase II strain, the phase I strain had lower growth yields and unexpectedly its spent media supported lower growth yields of $E$. coli, which might reflect the differential nutrient consumption by different C. burnetii strains. As the same inhibitor concentration in C. burnetii growth media maintains an E. coli-lethal effect during the 7-day incubation period and as C. burnetii $\mathrm{LpxC}$ is more susceptible to LPC-011 than E. coli LpxC (Figure 2), it is reasonable to conclude that the reduced growth phenotype of C. burnetii arises from $\mathrm{LpxC}$ inhibition and that lipid A biosynthesis is not essential for $C$. burnetii growth in axenic media.

\section{Regular Refreshment of LPC-011 Is Required for Consistent Inhibition of C. burnetii LpxC in Cell Cultures}

LPC-011 is a hydroxamic acid derivative. Compounds of the hydroxamate series were found to possess short pharmacokinetic half-lives when tested in vivo (Brown et al., 2012; Kalinin and Holl, 2017). Though LPC-011 has been tested for antibacterial activity in another obligate intracellular bacterium -Chlamydia (Nguyen et al., 2011; Cram et al., 2017), there is little information on the stability of LPC-011 in cell cultures. The chemical stability of LPC-011 is important in the context of the unique biology of C. burnetii -a 7-day growth cycle in acidic phagolysosomelike vacuoles. Therefore, we evaluated the stability of LPC011 in BGMK cell cultures by using growth of E. coli in cell culture medium as an indicator (Figure 4). BGMK cells can maintain a confluent monolayer without using FBS and were used in most of our C. burnetii infections. By collecting media every $24 \mathrm{~h}$ from mock or C. burnetii-infected cells and culturing E. coli TOP10 overnight, we found that a starting concentration of $2 \mu \mathrm{g} / \mathrm{mL}$ LPC-011 retained an $E$. coli-inhibiting concentration for at least $48 \mathrm{~h}$. Nonetheless, our data suggest that gradual depletion of the inhibitor was very likely due to the activity of BGMK cells, not C. burnetii. Thus, when using $2 \mu \mathrm{g} / \mathrm{mL}$ LPC-011 for testing its effectiveness of an antiC. burnetii agent, changing media containing freshly added inhibitors every $48 \mathrm{~h}$ was required to maintain an E. coliinhibiting concentration throughout the bacterium's growth cycle.

\section{LPC-011 Inhibits Lipid a Biosynthesis in a C. burnetii Phase II Strain Carrying C. trachomatis kdtA}

With the above established procedures of changing media containing $2 \mu \mathrm{g} / \mathrm{mL}$ LPC-011 every $48 \mathrm{~h}$, we investigated C. burnetii intracellular replication under conditions of $\mathrm{LpxC}$ inhibition. Whether lipid A biosynthesis of C. burnetii can be inhibited by LPC-011 under these conditions is unknown. We therefore established a lipid A reporter system to define the inhibition of lipid A biosynthesis in C. burnetii during infection of cultured cells. The C. burnetii phase II strain was 

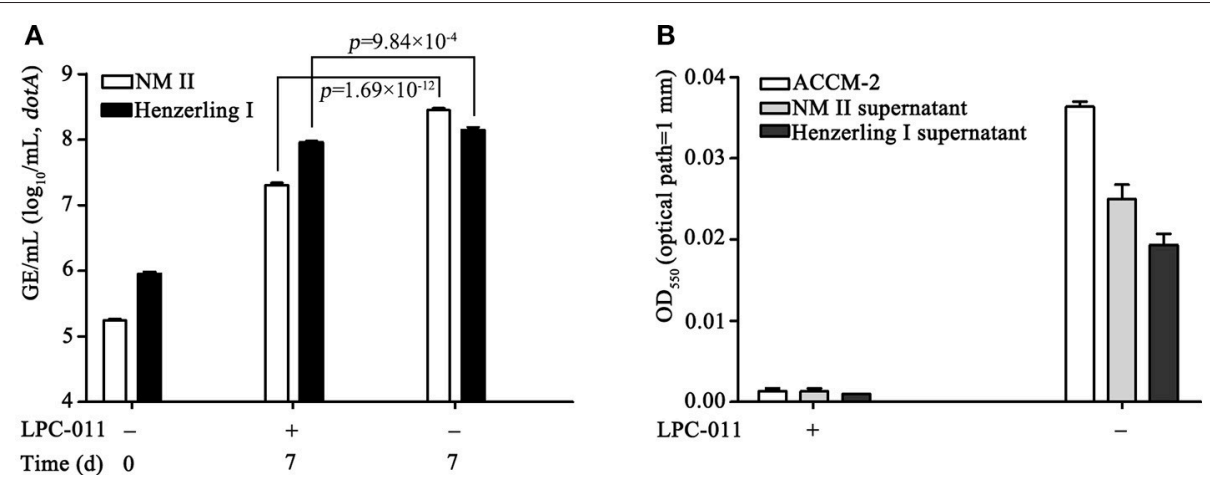

FIGURE 3 | Analysis of $C$. burnetii growth in axenic media containing a constant E. coli-lethal concentration of LPC-011. (A) Growth of $C$. burnetii Nine Mile phase II and Henzerling phase I in axenic media with or without $5 \mu \mathrm{g} / \mathrm{mL}$ LPC-011 were quantified by qPCR. (B) Overnight growth of $E$. coli TOP10 in axenic media with or without $5 \mu \mathrm{g} / \mathrm{mL}$ LPC-011 after $C$. burnetii inoculation for 7 days.

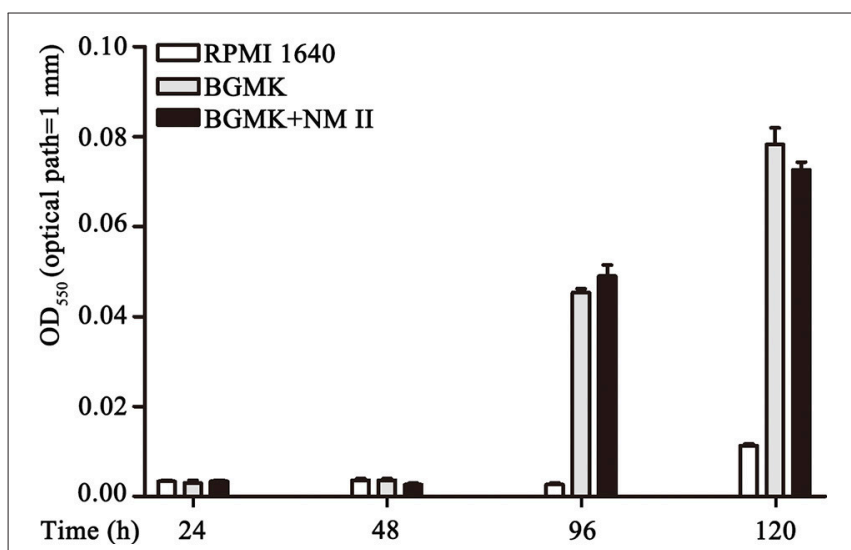

FIGURE 4 | Stability assessment of LPC-011 in C. burnetii cell culture. Overnight growth of E. coli in LPC-011-containing $(2 \mu \mathrm{g} / \mathrm{mL})$ cell media collected at different time points after incubation at $37^{\circ} \mathrm{C}, 5 \% \mathrm{CO}_{2}$ was measured. Three groups - media alone, normal cell culture, and C. burnetii infected cells were included.

transformed with a RSF1010 ori-based vector pMMGKkdtA that carries the $k d t A$ gene of Chlamydia trachomatis (Figure 5A). The chlamydial KdtA is a tri-functional Kdo transferase (Belunis et al., 1995) and can modify C. burnetii lipid A with a unique $\alpha$ Kdo- $(2 \rightarrow 8)-\alpha$-Kdo disaccharide, which can be detected by antiChlamydia genus-specific monoclonal antibodies (Figure 5B). The C. burnetii transformant (C. burnetii NM IIpMMGKkdtA, termed $C b: C t k d t A$ ) grew normally in BGMK cells. Its lipid A can be efficiently detected by immunofluorescence with antiChlamydia genus antibodies, indicating modification of lipid A as predicted (Figure 5B).

Under conditions of established LpxC inhibition in BGMK cells, the parent $C b: C t k d t A$ strain can form normal parasitophorous vacuoles though most vacuoles do not appear to be fully filled with progeny particles after 7 days of growth and growth yield was only about $0.5 \log$ (Figure 5C); importantly however, little or no lipid A could be detected by immunofluorescence with anti-Chlamydia antibodies. The $C b: C t k d t A$ strain was expanded in BGMK cultures under LpxC inhibition, then used to infect new cultures of BGMK and THP-1 cells. These progenies grew normally in BGMK cells without using inhibitors, but had fewer vacuoles and very limited growth yields in the presence of LPC-011. In macrophage-like THP-1 cells under similar conditions of LpxC inhibition, the parent $C b: C t k d t A$ strain had slow growth and vacuole expansion (data not shown), limited growth yield, and no lipid A could be detected. Moreover, the progenies prepared from BGMK cultures completely failed to form vacuoles upon re-infection in THP-1. However, in the absence of LPC-011, the same progenies were able to establish infection and grow normally in THP-1, suggesting lipid A is not involved in early vacuole development. Collectively, LPC-011 can effectively inhibit lipid A biosynthesis in the $C b: C t k d t A$ strain during infection of BGMK and THP-1 cells.

\section{Growth of Wild-Type C. burnetii Phase I and Phase II on BGMK Cells Under Constant LpxC Inhibition}

Having confirmed the inhibition of lipid A biosynthesis in cell cultures, we next compared the inhibitory effects of LPC011 between wild type phase I and phase II strains. Similar to results of our preliminary experiments, under conditions of LpxC inhibition in BGMK cells after 7 days post-infection, both phase I and phase II strains and their progenies prepared from inhibitor-treated BGMK cells appeared to form vacuoles mostly compacted with bacterial particles (Figure 6A), in contrast to the partially filled vacuoles of the $C b: C t k d t A$ strain. However, the inhibitor does affect growth yields of both parent strains and their progenies $(p<0.05)$ (Figure 6B, Table S2), which is at least in part due to their reduced vacuole numbers (Figure S1). The reduced number of productive vacuoles due to the inhibitor is particularly significant in low MOI infections, e.g. about 50\% less vacuoles were formed in MOI $=1$ infections of both strains (Figure S1). To be noted, the active Brownian movement of evenly distributed bacterial particles of the phase I strain inside 

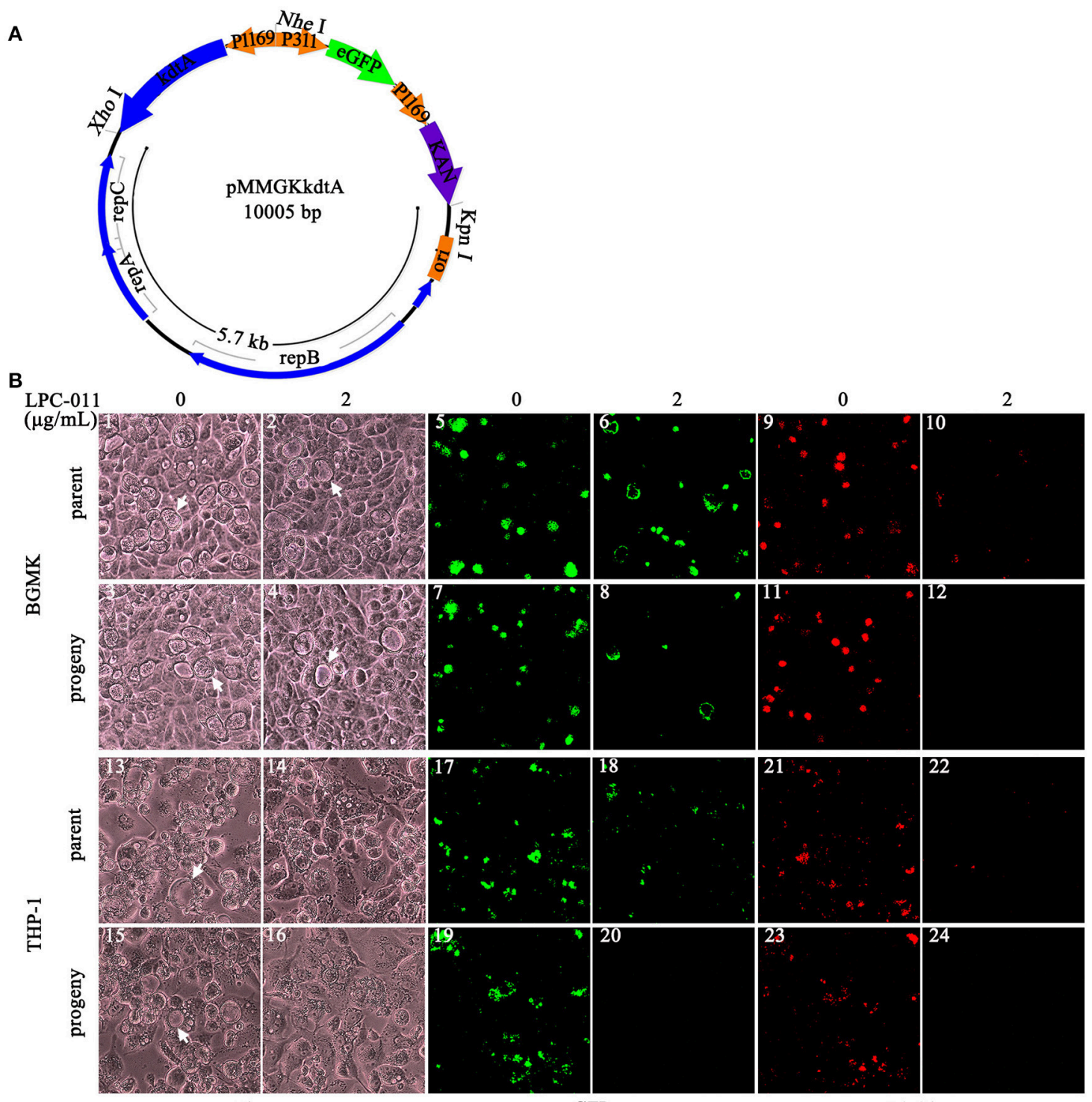

C

Phase

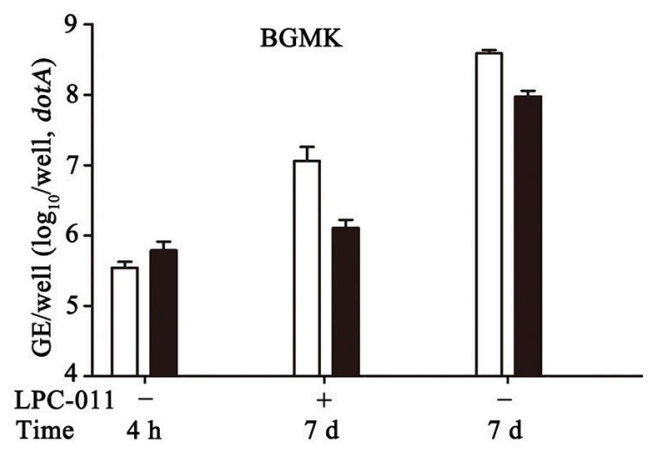

GFP

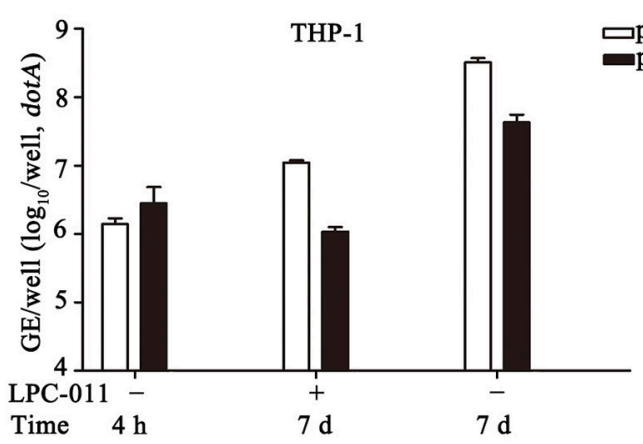

FIGURE 5 | LPC-011 inhibits lipid A biosynthesis in Cb:Ct kdtA (a C. burnetii transformant carrying C. trachomatis kdtA). (A) Plasmid map of pMMGKkdtA. It has a $5.7 \mathrm{~kb}$ segment from pMMB207, chlamydial $k d t A$ and two marker genes (eGFP and Kan ${ }^{\mathrm{B}}$ ). (B) Growth of $C$. burnetii (parent $C b: C t$ kdtA and its progeny prepared from LPC-011 treated BGMK cells) on BGMK (1-12) and THP-1 (13-24) cells with or without LPC-011 treatment. Vacuole and bacterial growth, and lipid A expression was assessed by phase microscopy, observing eGFP expression, and immunofluorescence, respectively, after $7 \mathrm{~d}$ growth. Arrows indicate CCVs. (C) Quantitative analysis of $C$. burnetii (parent $C b: C t$ kdtA and its progeny prepared from LPC-011 treated BGMK cells) growth on BGMK and THP-1 cells with or without LPC-011 treatment. 

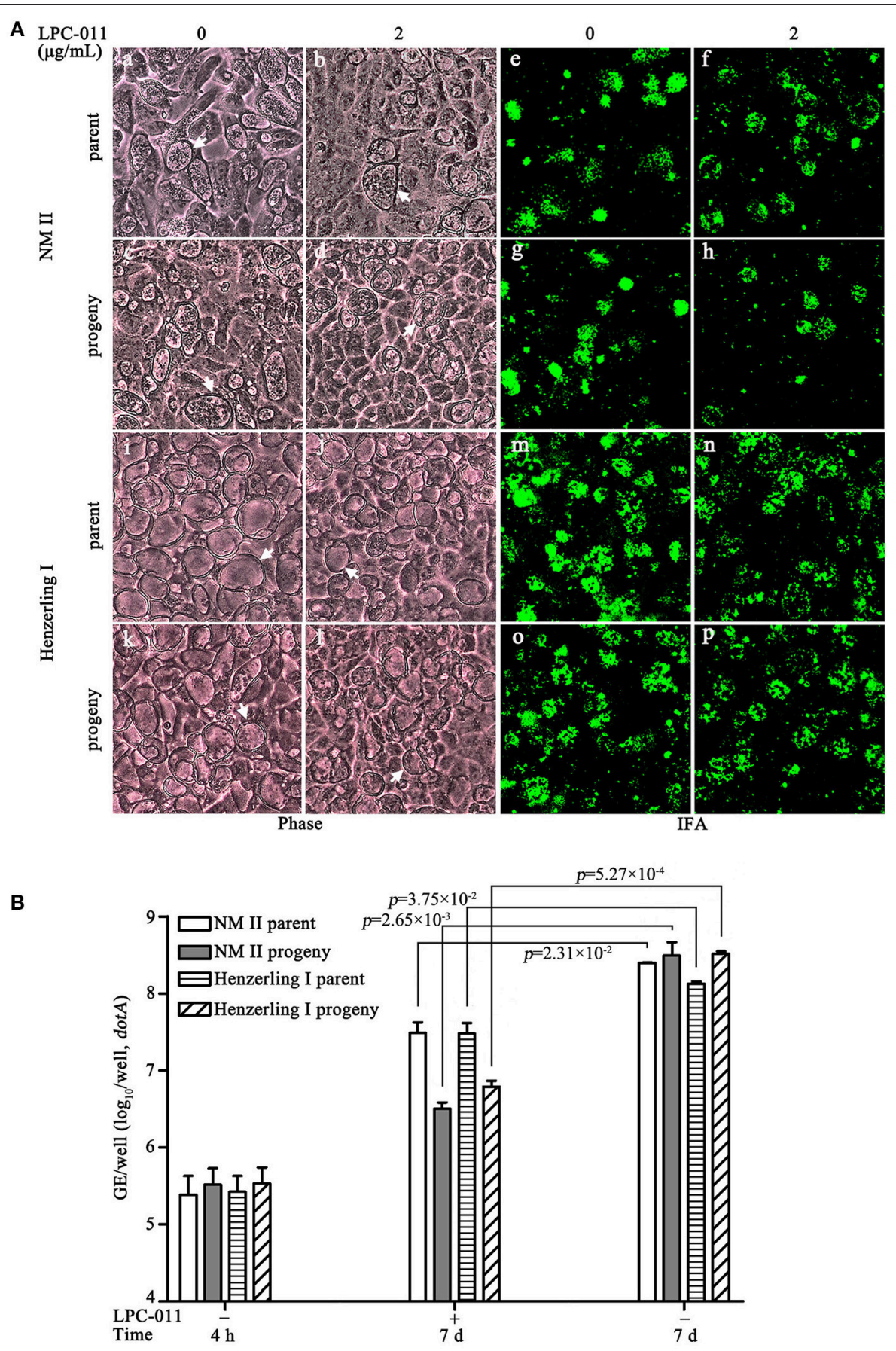

FIGURE 6 | Growth of wild type C. burnetii in inhibitor-treated BGMK cells. (A) C. burnetii growth in BGMK cells with or without inhibitor treatment was assessed by phase contrast microcopy and immunofluorescence. Two C. burnetii strains -Nine Mile phase II and Henzerling phase I and their progenies prepared from inhibitor-treated BGMK cells were included. Arrows indicate CCVs. (B) Growth yield analysis of the above two C. burnetii strains in BGMK cells with or without LPC-011.

the vacuoles was not affected by LPC-011, suggesting that LPS is not the only factor contributing to high hydrophilicity of the phase I strain (data not shown).

In Chlamydia trachomatis, LPC-011 can block the transition from replicative reticulate body to infectious elementary body forms (Nguyen et al., 2011). Inhibiting lipid A biosynthesis might also relate to the structural transition of $C$. burnetii, though its two structural forms are both infectious, as indicated by the reduced number of productive vacuoles. Thus, we performed an ultrastructural analysis by transmission electron microscopy 
(TEM) to assess the developmental transitions (Figure 7). A portion of SCV-sized $(0.2-0.5 \mu \mathrm{m})$ bodies can be found in vacuoles of inhibitor-treated cells 7 days post-infection. Whether these SCV-sized bodies express protein markers specific to the SCV morphotype is unknown. The TEM data suggests lipid $\mathrm{A}$ is not required for the morphological transition of LCV to SCV. Altogether, inhibiting lipid A biosynthesis has moderate effects on survival and growth of wild type phase I and phase II C. burnetii strains in BGMK cells.

\section{Limited Propagation of C. burnetii in LPC-011-Treated THP-1 Cells}

The $C b: C t k d t A$ strain, especially its progeny prepared with inhibitor treatment, has severe growth defects in THP-1. Testing growth of wild type C. burnetii in inhibitor-treated macrophages is more relevant for evaluating both the inhibitor's therapeutic use for curing $\mathrm{Q}$ fever and the role of lipid A in C. burnetii biology. In macrophage-like THP-1 cells with LPC-011 treatment, wild type phase I and phase II C. burnetii strains, and their progenies prepared from inhibitor-treated BGMK cultures show similar growth defects (Figure 8). They all appear to form small vacuoles albeit with an occurrence of normal size vacuoles, and all their growth yields increased $<0.5$ $\log$ (Table S2). Compared to the respective genome copies at $4 \mathrm{~h}$ post-infection, the growth yield of progenies of Nine Mile phase II under inhibitor treatment only showed a trend toward enhanced progeny ( $p=0.1$ ), while growth yields of other three C. burnetii preparations reached statistical significance $(p<0.05)$ (Figure 8B, Table S2). All four C. burnetii preparations also appear to form a reduced number of vacuoles, but we did not quantify their small vacuoles due to enumerating difficulties. In addition, no mature vacuoles filled with $C$. burnetii particles as in cells without inhibitor-treatment can be observed by phase contrast microscopy. Clearly, lipid A plays a more important role for $C$. burnetii survival and growth in macrophages, which was also suggested by the $C b: C t k d t A$ strain.

\section{DISCUSSION}

LPS is a highly conserved component in the outer membrane of Gram-negative bacteria. Pioneering work in E. coli and Salmonella established that lipid A and Kdo components of LPS are essential for outer membrane biogenesis and cell viability. However, with the isolation of LPS-deficient mutants of Neisseria meningitidis, Moraxella catarrhalis, and Acinetobacter baumannii, it has become clear that the essentiality of Kdo-lipid A of Gram-negative bacteria varies considerably, depending on the species and even on the particular strain background (Bos and Tommassen, 2005; Peng et al., 2005; Moffatt et al., 2010; Zhang et al., 2013). Here we provide evidence that lipid A is nonessential for C. burnetii survival and growth in vitro, and lipid A has variable roles for bacterial robust growth in different culture systems. Lipid A has significance for optimal replication in macrophages, but appears less significant for replication in non-phagocytic epithelial cells and culture in axenic media.

The ACCM culture techniques greatly promote the development of new methods and tools for C. burnetii research (Beare, 2012; Omsland, 2012; Beare and Heinzen, 2014). In this case the ACCM techniques will likely aid the construction of $l_{p x C}$ null mutants. C. burnetii is an obligate intracellular bacterium in nature. C. burnetii genes or factors that are dispensable in axenic culture, could play important roles in vivo. For example, the type IV secretion system of C. burnetii is found to be required for the development of mature CCV (Beare et al., 2011; Carey et al., 2011). In addition, for unknown reasons, the viability of stationary-phase SCV propagated in ACCM-2 is substantially less than that of host cell-propagated SCVs (Sandoz et al., 2016). Thus we prepared all our strains from BGMK cultures and focused on the role of lipid A in C. burnetii growth in various cell lines.

C. burnetii preferentially infects mononuclear phagocytes, such as alveolar macrophages (Howe et al., 2010). Phagocytes
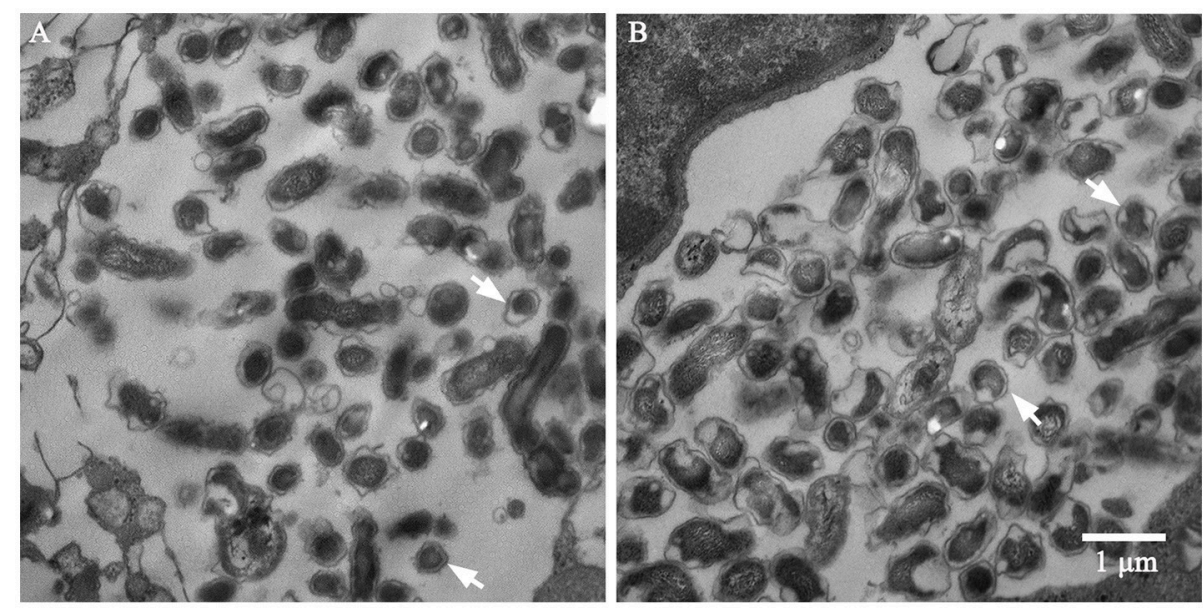

FIGURE 7 | TEM analysis of C. burnetii Nine Mile phase II in BGMK cells with (A) or without (B) inhibitor treatment 7 days post-infection. Bacterial bodies of SCV size can be found in both vacuoles. Arrows indicate SCVs. Scale bar is $1 \mu \mathrm{m}$. 


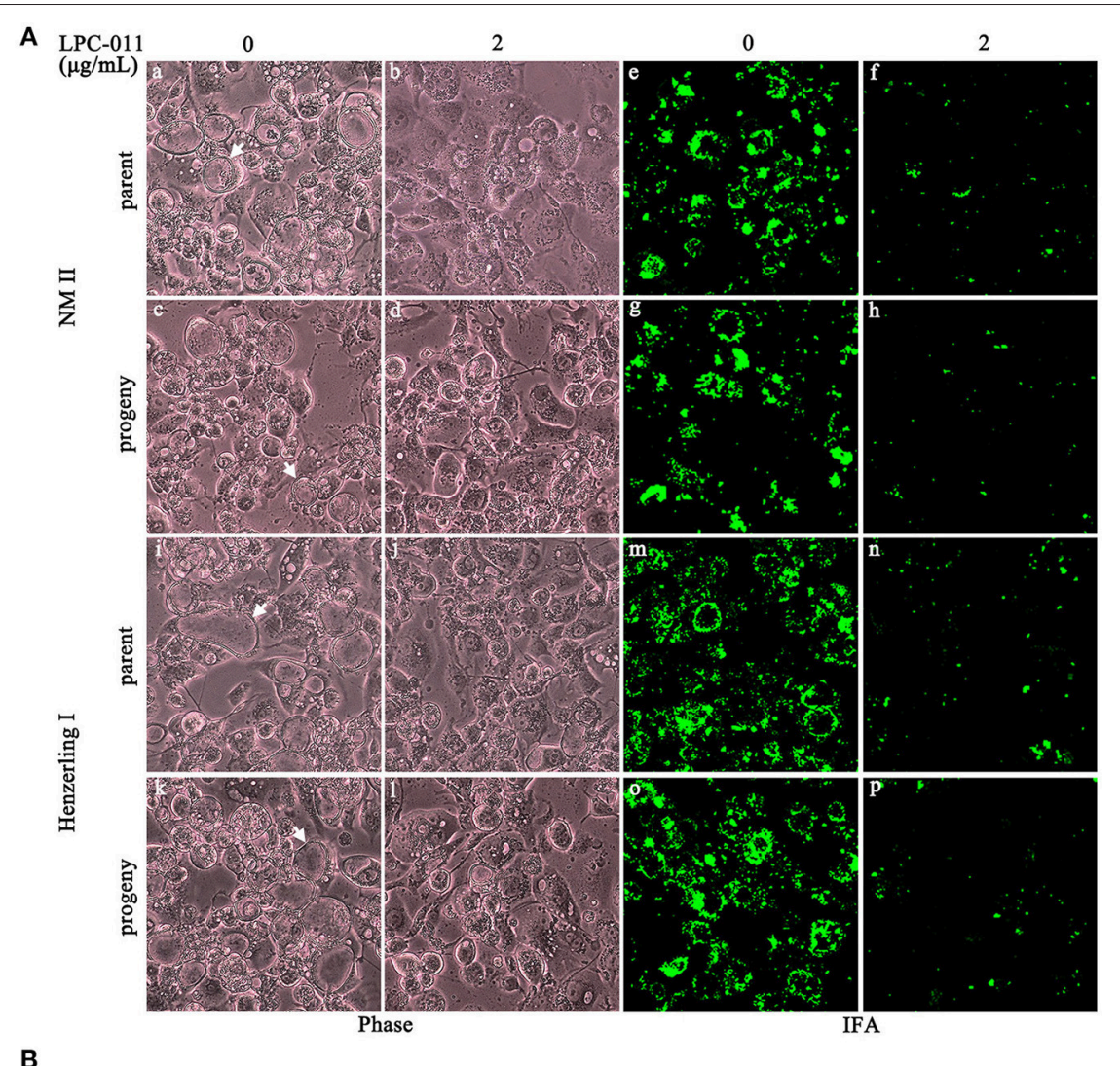

B

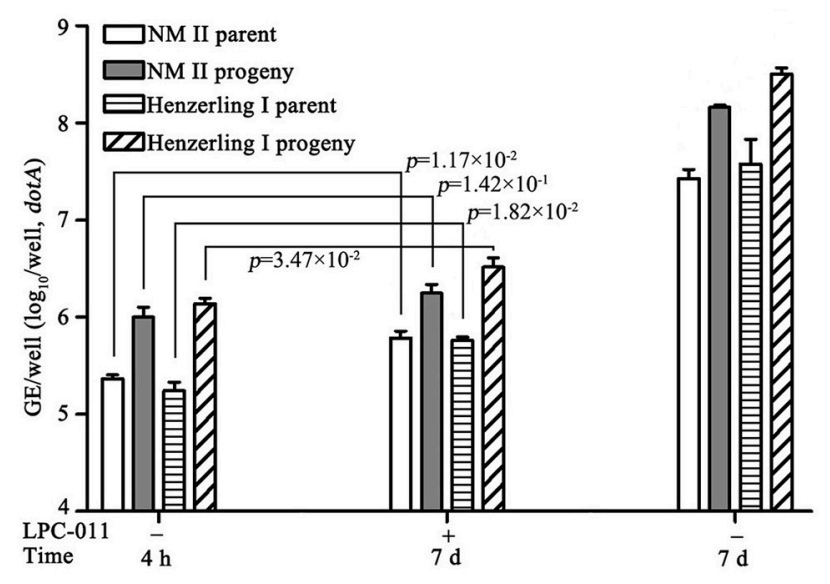

FIGURE 8 | Growth of wild type C. burnetii in inhibitor-treated THP-1 cells. (A) Four C. burnetii strains-Nine Mile phase II, Henzerling phase I and their progenies prepared from inhibitor-treated BGMK cells, were used to infect differentiated THP-1 cells with or without inhibitor treatment. Their growths were assessed by phase microcopy and immunofluorescence. Arrows indicate CCVs. (B) Growth yields of the above infections on THP-1 were quantified by qPCR.

have a repertoire of antimicrobial or bactericidal mechanisms. In the more relevant macrophage-like THP-1 cells with LpxC inhibitor treatment, C. burnetii formed small vacuoles and exhibited limited growth. The role of lipid A for both optimal vacuole formation and bacterial growth of C. burnetii in THP1 cells likely reflects some unique adaptations of the organism to resist the antimicrobial functions of its phagolysosome-like CCV. Our findings highlight the significance of using more relevant cells for investigating the importance of lipid A in intracellular pathogens. In macrophage-targeted Chlamydia trachomatis LGV biovar L2 434/Bu, lipid A was found to be required for generating infectious particles, but had no effect on either vacuole development or bacterial replication in nonrelevant epithelial cells (Nguyen et al., 2011). It would be of interest to determine whether lipid A of C. trachomatis LGV biovar has similar roles in resisting antimicrobial activities of macrophages.

The CCV is a unique niche for $C$. burnetii replication. The maturation of the CCV is a process orchestrated by type IV 
secretion system and its effectors (Ghigo et al., 2012; Kohler and Roy, 2015). The variable roles of lipid A for C. burnetii growth in different cell types coincide with the distinct CCV growth defects under inhibitor treatment. In Gram-negative bacteria, inhibition of lipid A biosynthesis could compromise some essential functions of the cell envelope, such as affecting the assembly and function of membrane proteins (Zhang et al., 2013). While the growth of most CCVs in epithelial cells is not apparently affected, the reduced number of mature CCVs in epithelial cells and the near absence of mature CCVs in phagocytes may reflect the variable essentiality of secreted effectors that are affected by the loss of lipid A. Future experiments will aim at deleting the lp $x$ C gene from phase I and phase II C. burnetii and determining the physiological rebalance and respective features of both SCV and LCV upon losing lipid A.

CCVs of phase I and phase II C. burnetii strains exhibit distinct late-growth morphological features in epithelial cells. Bacterial particles within phase II Nine Mile CCVs have little intravacuolar movement, while particles within phase I Henzerling CCVs exhibit characteristic Brownian-like movement. This contrasting movement feature may be due to the distinct LPS structures of phase I and phase II particles, as shortened LPS is assumed to increase the hydrophobicity of phase II strains. However, it is unexpected that the distinct movement features of phase I and phase II strains were not affected by inhibiting lipid A biosynthesis. Our data suggest that besides the full length LPS, other surface structures like glycosylated proteins might also contribute to the hydrophilicity of phase I strains. Clearly, further experimentation is needed to determine whether LPS biosynthesis in phase I strain infected cells is effectively depleted by the inhibitors.

Antibiotic susceptibility testing of $C$. burnetii is difficult because this organism is a fastidious and slow-growing intracellular bacterium. A number of cell lines such as P388D1 and J774 (murine macrophage-like cell lines), L929 (a murine fibroblast cell line), and HEL (human embryonic lung cells), have been used to test antibiotic activity against C. burnetii (Angelakis and Raoult, 2010). Clearly, our findings also indicate the importance of using more relevant cells for testing antibiotic efficacies against intracellular pathogens. In the case of C. burnetii, new clinical therapies for chronic Q fever are needed (Mulye et al., 2017). Our finding of profound inhibition of $C$. burnetii growth in THP-1 cells by LpxC

\section{REFERENCES}

Amano, K., Williams, J. C., Missler, S. R., and Reinhold, V. N. (1987). Structure and biological relationships of Coxiella burnetii lipopolysaccharides. J. Biol. Chem. 262, 4740-4747.

Andoh, M., Zhang, G., Russell-Lodrigue, K. E., Shive, H. R., Weeks, B. R., and Samuel, J. E. (2007). T cells are essential for bacterial clearance, and gamma interferon, tumor necrosis factor alpha, and B cells are crucial for disease development in Coxiella burnetii infection in mice. Infect. Immun. 75, 3245-3255. doi: 10.1128/IAI.01767-06

Angelakis, E., and Raoult, D. (2010). Q Fever. Vet. Microbiol. 140, 297-309. doi: 10.1016/j.vetmic.2009.07.016

Barb, A. W., and Zhou, P. (2008). Mechanism and inhibition of LpxC: an essential zinc-dependent deacetylase of bacterial lipid A synthesis. Curr. Pharm. Biotechnol. 9, 9-15. doi: 10.2174/138920108783497668 inhibitors encourages additional analysis of the use of these inhibitors as potential therapeutics for the treatment of $Q$ fever.

\section{AUTHOR CONTRIBUTIONS}

TW, YY, SL, ZH, ZS, and YJ: data acquisition, data analysis, data interpretation, revising of the manuscript; XL, AO, and $\mathrm{PZ}$ : reagent provision, data analysis, data interpretation, revising of the manuscript; TW and LS: data acquisition, data analysis, data interpretation, writing of the manuscript, revising of the manuscript.

\section{FUNDING}

This work was supported by National Key R\&D Program of China (No. 2016YFC1202705), National Natural Science Foundation of China (No. 31570177), and Foundation of State Key Laboratory of Pathogen and Biosecurity (No. SKLPBS1409). The synthesis of LpxC inhibitors was supported by a grant to PZ (GM115355) from the National Institute of General Medical Sciences, USA.

\section{ACKNOWLEDGMENTS}

We thank Dr. Xuehong Zhang from Shanghai Jiaotong University for providing the pMMB207 plasmid.

\section{SUPPLEMENTARY MATERIAL}

The Supplementary Material for this article can be found online at: https://www.frontiersin.org/articles/10.3389/fcimb. 2018.00192/full\#supplementary-material

Datasheet S1 | Complete sequence of the pMMGKkdtA plasmid.

Figure S1 | CCV enumeration in C. burnetii $(\mathrm{MOI}=1)$ infected BGMK cells with or without inhibitor treatment. (A) Representative images of phase contrast microscopy of $C$. burnetii infected BGMK cells with or without inhibitor treatment. Two C. burnetii strains -Nine Mile phase II and Henzerling phase I and their progenies prepared from inhibitor-treated BGMK cells were included. Arrows indicate CCVs. (B) Quantitative analysis of CCV numbers formed by different C. burnetii strains.

Table S1 | Oligonucleotides used in this study.

Table S2 | qPCR analyses of $C$. burnetii growth in different culture systems with or without inhibitor treatment.

Beare, P. A. (2012). Genetic manipulation of Coxiella burnetii. Adv. Exp. Med. Biol. 984, 249-271. doi: 10.1007/978-94-007-4315-1_13

Beare, P. A., and Heinzen, R. A. (2014). Gene inactivation in Coxiella burnetii. Methods Mol. Biol. 1197, 329-345. doi: 10.1007/978-1-4939-1261-2_19

Beare, P. A., Gilk, S. D., Larson, C. L., Hill, J., Stead, C. M., Omsland, A., et al. (2011). Dot/Icm type IVB secretion system requirements for Coxiella burnetii growth in human macrophages. MBio 2, e00175-e00111. doi: 10.1128/mBio.00175-11

Belunis, C. J., Clementz, T., Carty, S. M., and Raetz, C. R. (1995). Inhibition of lipopolysaccharide biosynthesis and cell growth following inactivation of the kdtA gene in Escherichia coli. J. Biol. Chem. 270, 27646-27652. doi: $10.1074 /$ jbc. 270.46 .27646

Bos, M. P., and Tommassen, J. (2005). Viability of a capsule- and lipopolysaccharide-deficient mutant of Neisseria meningitidis. Infect. Immun. 73, 6194-6197. doi: 10.1128/IAI.73.9.6194-6197.2005 
Brown, M. F., Reilly, U., Abramite, J. A., Arcari, J. T., Oliver, R., Barham, R. A., et al. (2012). Potent inhibitors of LpxC for the treatment of Gram-negative infections. J. Med. Chem. 55, 914-923. doi: 10.1021/jm2014748

Carey, K. L., Newton, H. J., Luhrmann, A., and Roy, C. R. (2011). The Coxiella burnetii Dot/Icm system delivers a unique repertoire of type IV effectors into host cells and is required for intracellular replication. PLoS Pathog. 9: e1002056. doi: 10.1371/journal.ppat.1002056

Chung, H. S., and Raetz, C. R. (2011). Dioxygenases in Burkholderia ambifaria and Yersinia pestis that hydroxylate the outer Kdo unit of lipopolysaccharide. Proc. Natl. Acad. Sci. U.S.A. 108, 510-515. doi: 10.1073/pnas.1016462108

Coggins, B. E., Li, X., Mcclerren, A. L., Hindsgaul, O., Raetz, C. R., and Zhou, P. (2003). Structure of the LpxC deacetylase with a bound substrate-analog inhibitor. Nat. Struct. Biol. 10, 645-651. doi: 10.1038/nsb948

Coleman, S. A., Fischer, E. R., Howe, D., Mead, D. J., and Heinzen, R. A. (2004). Temporal analysis of Coxiella burnetii morphological differentiation. J. Bacteriol. 186, 7344-7352. doi: 10.1128/JB.186.21.7344-7352.2004

Cram, E. D., Rockey, D. D., and Dolan, B. P. (2017). Chlamydia spp. development is differentially altered by treatment with the LpxC inhibitor LPC-011. BMC Microbiol. 17:98. doi: 10.1186/s12866-017-0992-8

Ghigo, E., Colombo, M. I., and Heinzen, R. A. (2012). The Coxiella burnetii parasitophorous vacuole. Adv. Exp. Med. Biol. 984, 141-169. doi: 10.1007/978-94-007-4315-1_8

Hackstadt, T. (1988). Steric hindrance of antibody binding to surface proteins of Coxiella burnetti by phase I lipopolysaccharide. Infect. Immun. 56, 802-807.

Harris, R. J., Storm, P. A., Lloyd, A., Arens, M., and Marmion, B. P. (2000). Long-term persistence of Coxiella burnetii in the host after primary Q fever. Epidemiol. Infect. 124, 543-549. doi: 10.1017/S0950268899003763

Hechemy, K. E. (2012). History and prospects of Coxiella burnetii research. Adv. Exp. Med. Biol. 984, 1-11. doi: 10.1007/978-94-007-4315-1_1

Howe, D., Shannon, J. G., Winfree, S., Dorward, D. W., and Heinzen, R. A. (2010). Coxiella burnetii phase I and II variants replicate with similar kinetics in degradative phagolysosome-like compartments of human macrophages. Infect. Immun. 78, 3465-3474. doi: 10.1128/IAI.00406-10

Kalinin, D. V., and Holl, R. (2017). LpxC inhibitors: a patent review (2010-2016). Expert Opin. Ther. Pat. 27, 1227-1250. doi: 10.1080/13543776.2017.1360282

Kohler, L. J., and Roy, C. R. (2015). Biogenesis of the lysosome-derived vacuole containing Coxiella burnetii. Microbes Infect. 17, 766-771. doi: 10.1016/j.micinf.2015.08.006

Larson, C. L., Beare, P. A., Voth, D. E., Howe, D., Cockrell, D. C., Bastidas, R. J., et al. (2015). Coxiella burnetii effector proteins that localize to the parasitophorous vacuole membrane promote intracellular replication. Infect. Immun. 83, 661-670. doi: 10.1128/IAI.02763-14

Larson, C. L., Martinez, E., Beare, P. A., Jeffrey, B., Heinzen, R. A., and Bonazzi, M. (2016). Right on Q: genetics begin to unravel Coxiella burnetii host cell interactions. Future Microbiol. 11, 919-939. doi: 10.2217/fmb2016-0044

Lee, C. J., Liang, X., Chen, X., Zeng, D., Joo, S. H., Chung, H. S., et al. (2011). Species-specific and inhibitor-dependent conformations of LpxC: implications for antibiotic design. Chem. Biol. 18, 38-47. doi: 10.1016/j.chembiol.2010.11.011

Liang, X., Lee, C. J., Chen, X., Chung, H. S., Zeng, D., Raetz, C. R., et al. (2011). Syntheses, structures and antibiotic activities of LpxC inhibitors based on the diacetylene scaffold. Bioorg. Med. Chem. 19, 852-860. doi: 10.1016/j.bmc.2010.12.017

Maurin, M., and Raoult, D. (1999). Q fever. Clin. Microbiol. Rev. 12, 518-553.

Minnick, M. F., and Raghavan, R. (2012). Developmental biology of Coxiella burnetii. Adv. Exp. Med. Biol. 984, 231-248. doi: 10.1007/978-94-007-4315-1_12

Moffatt, J. H., Harper, M., Harrison, P., Hale, J. D., Vinogradov, E., Seemann, T., et al. (2010). Colistin resistance in Acinetobacter baumannii is mediated by complete loss of lipopolysaccharide production. Antimicrob. Agents Chemother. 54, 4971-4977. doi: 10.1128/AAC.00834-10

Moos, A., and Hackstadt, T. (1987). Comparative virulence of intra- and interstrain lipopolysaccharide variants of Coxiella burnetii in the guinea pig model. Infect. Immun. 55, 1144-1150.

Mulye, M., Samanta, D., Winfree, S., Heinzen, R. A., and Gilk, S. D. (2017). Elevated cholesterol in the Coxiella burnetii intracellular niche is bacteriolytic. MBio 8, e02313-e02316. doi: 10.1128/mBio.02313-16
Narasaki, C. T., and Toman, R. (2012). Lipopolysaccharide of Coxiella burnetii. Adv. Exp. Med. Biol. 984, 65-90. doi: 10.1007/978-94-007-4315-1_4

Nguyen, B. D., Cunningham, D., Liang, X., Chen, X., Toone, E. J., Raetz, C. R., et al. (2011). Lipooligosaccharide is required for the generation of infectious elementary bodies in Chlamydia trachomatis. Proc. Natl. Acad. Sci. U.S.A. 108, 10284-10289. doi: 10.1073/pnas.1107478108

Omsland, A. (2012). Axenic growth of Coxiella burnetii. Adv. Exp. Med. Biol. 984, 215-229. doi: 10.1007/978-94-007-4315-1_11

Omsland, A., Beare, P. A., Hill, J., Cockrell, D. C., Howe, D., Hansen, B., et al. (2011). Isolation from animal tissue and genetic transformation of Coxiella burnetii are facilitated by an improved axenic growth medium. Appl. Environ. Microbiol. 77, 3720-3725. doi: 10.1128/AEM.02826-10

Omsland, A., Cockrell, D. C., Howe, D., Fischer, E. R., Virtaneva, K., Sturdevant, D. E., et al. (2009). Host cell-free growth of the Q fever bacterium Coxiella burnetii. Proc. Natl. Acad. Sci. U.S.A. 106, 4430-4434. doi: 10.1073/pnas.0812074106

Peng, D., Hong, W., Choudhury, B. P., Carlson, R. W., and Gu, X. X. (2005). Moraxella catarrhalis bacterium without endotoxin, a potential vaccine candidate. Infect. Immun. 73, 7569-7577. doi: 10.1128/IAI.73.11.7569-7577.2005

Raetz, C. R., Reynolds, C. M., Trent, M. S., and Bishop, R. E. (2007). Lipid A modification systems in gram-negative bacteria. Annu. Rev. Biochem. 76, 295-329. doi: 10.1146/annurev.biochem.76.010307.145803

Sandoz, K. M., Beare, P. A., Cockrell, D. C., and Heinzen, R. A. (2016). Complementation of arginine auxotrophy for genetic transformation of Coxiella burnetii by use of a defined axenic medium. Appl. Environ. Microbiol. 82, 3042-3051. doi: 10.1128/AEM.00261-16

Shannon, J. G., Howe, D., and Heinzen, R. A. (2005). Virulent Coxiella burnetii does not activate human dendritic cells: role of lipopolysaccharide as a shielding molecule. Proc. Natl. Acad. Sci. U.S.A. 102, 8722-8727. doi: 10.1073/pnas.0501863102

Toman, R., and Skultety, L. (1994). Analysis of the 3-deoxy-D-manno-2octulosonic acid region in a lipopolysaccharide isolated from Coxiella burnetii strain Nine Mile in phase II. Acta. Virol. 38, 241-243.

Tomaras, A. P., Mcpherson, C. J., Kuhn, M., Carifa, A., Mullins, L., George, D., et al. (2014). LpxC inhibitors as new antibacterial agents and tools for studying regulation of lipid A biosynthesis in Gram-negative pathogens. MBio 5:e01551-e01514. doi: 10.1128/mBio.01551-14

Vallejo Esquerra, E., Yang, H., Sanchez, S. E., and Omsland, A. (2017). Physicochemical and nutritional requirements for axenic replication suggest physiological basis for Coxiella burnetii niche restriction. Front. Cell. Infect. Microbiol. 7:190. doi: 10.3389/fcimb.2017.00190

Waag, D. M. (2007). Coxiella burnetii: host and bacterial responses to infection. Vaccine 25, 7288-7295. doi: 10.1016/j.vaccine.2007.08.002

Whittington, D. A., Rusche, K. M., Shin, H., Fierke, C. A., and Christianson, D. W. (2003). Crystal structure of LpxC, a zinc-dependent deacetylase essential for endotoxin biosynthesis. Proc. Natl. Acad. Sci. U.S.A. 100, 8146-8150. doi: 10.1073/pnas.1432990100

Wiebe, M. E., Burton, P. R., and Shankel, D. M. (1972). Isolation and characterization of two cell types of Coxiella burneti phase I. J. Bacteriol. 110, 368-377.

Zhang, G., Meredith, T. C., and Kahne, D. (2013). On the essentiality of lipopolysaccharide to Gram-negative bacteria. Curr. Opin. Microbiol. 16, 779-785. doi: 10.1016/j.mib.2013.09.007

Zhou, P., and Zhao, J. (2017). Structure, inhibition, and regulation of essential lipid A enzymes. Biochim. Biophys. Acta 1862, 1424-1438. doi: 10.1016/j.bbalip.2016.11.014

Conflict of Interest Statement: The authors declare that the research was conducted in the absence of any commercial or financial relationships that could be construed as a potential conflict of interest.

Copyright (C) 2018 Wang, Yu, Liang, Luo, He, Sun, Jiang, Omsland, Zhou and Song. This is an open-access article distributed under the terms of the Creative Commons Attribution License (CC BY). The use, distribution or reproduction in other forums is permitted, provided the original author(s) and the copyright owner are credited and that the original publication in this journal is cited, in accordance with accepted academic practice. No use, distribution or reproduction is permitted which does not comply with these terms. 